\title{
Deep Learning Power of TOR: Security Levels
}

\author{
V Prabhavathi ${ }^{1}$, Dr S Pradeep ${ }^{2}$, Kumar Swamy $^{3}$, A Damdor ${ }^{4}$ \\ ${ }^{1}$ Assistant professor, Dept of CSE, Malla Reddy Engineering College for Women \\ ${ }^{2}$ Assistant professor, Dept of CSE, Malla Reddy Engineering College for Women \\ ${ }^{3}$ Assistant professor, Dept of CSE, Malla Reddy Engineering College for Women \\ 4Assistant professor, Dept of CSE, Malla Reddy Engineering College for Women \\ (UGC Autonomous Campus) Maisammguda, Secunderabad, Telangana, India. \\ 1prabhag9@gmail.com ,2pradeep.sunkari87@gmail.com \\ ${ }^{3}$ Kankala.kumar24@gmail.com ${ }^{4}$ damodardaniel@gmail.com
}

\begin{abstract}
Most of the profound learning applications that we find locally are typically outfitted towards fields like advertising, deals, finance, and so on We scarcely at any point read articles or discover assets about profound getting the hang of being utilized to secure these items, and the business, from malware and programmer assaults. While the enormous innovation organizations like Google, Facebook, Microsoft, and Sales force have effectively implanted profound learning into their items, the online protection industry is as yet playing make up for lost time. It's a difficult field however one that needs our complete consideration. we momentarily present Deep Learning $(D L)$ alongside a couple of existing Information Security (therefore alluded to as Information security analysts ) applications it empowers. We then, at that point profound plunge into the intriguing issue of unknown pinnacle traffic discovery and furthermore present a DL-based answer for distinguish TOR traffic.
\end{abstract}

Keywords: TOR Traffic, FFNN, Signature based, Anonymous Network Traffic, ML \& DL

\section{INTRODUCTION}

Profound learning is certifiably for data augmentation shot that can tackle all the information security analysts issues since it needs broad marked datasets. Lamentably, no such named datasets are promptly accessible. Notwithstanding, here we have a few systems uses situations where the profound learning networks are making huge enhancements to the current arrangements. Unintentional location and organization interruption recognition are two such regions where profound learning has shown critical enhancements over the standard based and exemplary AI based arrangements.

Organization interruption location frameworks are commonly procedure oriented and monogram controls that are conveyed at the edge to identify known dangers. Enemies change the malware marks and effectively dodge the conventional organization interruption identification frameworks. Quamar et al. [1], in the previous exchange paper, showed profound learning (DL)- based frameworks utilizing self-educated figuring out how to be promising in distinguishing obscure organization interruptions. Customary security use cases, for example, malware identification and spyware location have been handled with profound neural net-based frameworks [2].

The speculation force of DL-based procedures is better contrasted with conventional MLbased methodologies. Jung et al's. [3] DL based framework can even identify zero-day malware. Daniel Gibert [2], a Ph.D. move on from the University of Barcelona, has accomplished broad business related to convolutional neural organizations $(\mathrm{CNN}$, a kind of DL design) and malware discovery. In this research proposal, we say that CNNs can recognize even polymorphic malware. 
The DL-based neural nets are presently getting utilized in User and Entity Behavior Analytics (UEBA). Generally, UEBA utilizes inconsistency discovery and AI calculations which distil the security occasions to profile and pattern each client and organization component in the endeavor IT climate. Any huge deviations from the baselines were set off as oddities that further raised cautions to be explored by the security investigators. UEBA upgraded the location of insider dangers, though partially.

Presently, profound learning-based frameworks are utilized to identify numerous different kinds of irregularities. Paweł Kobojek from Warsaw college, Poland [4] utilizes keystroke elements to confirm the client utilizing a LSTM organization. Jason Trost, head of safety information designing at Capital One, has distributed a few websites [5] that have a rundown of specialized papers and chats on applying profound learning in system.

\section{RELATED WORK}

\subsection{Feed Forward Neural Network}

The fake neural organization is enlivened from the natural neural organization. Neurons are the nuclear unit of an organic neural organization. Every neuron comprises of dendrites, core, and axons. It gets signals through dendrites and is brought out through axons. The calculations are acted in the core. The whole organization is comprised of a neural circuit interconnected by synapses.

Computer based intelligence analysts acquired this plan to foster the fake neural organization (ANN). In this setting, every neuron achieves three activities:

a. This gathers input from different neurons or contributions to a weighted way

b. This summarizes all info signals

c. It will light of the added esteem, it calls an actuation work

Every neuron subsequently can arrange whether a bunch of data sources have a place with some class. It forces is restricted when just a solitary neuron is utilized. Be that as it may, begetting a bunch of neurons makes it an amazing apparatus for grouping and succession naming undertakings.
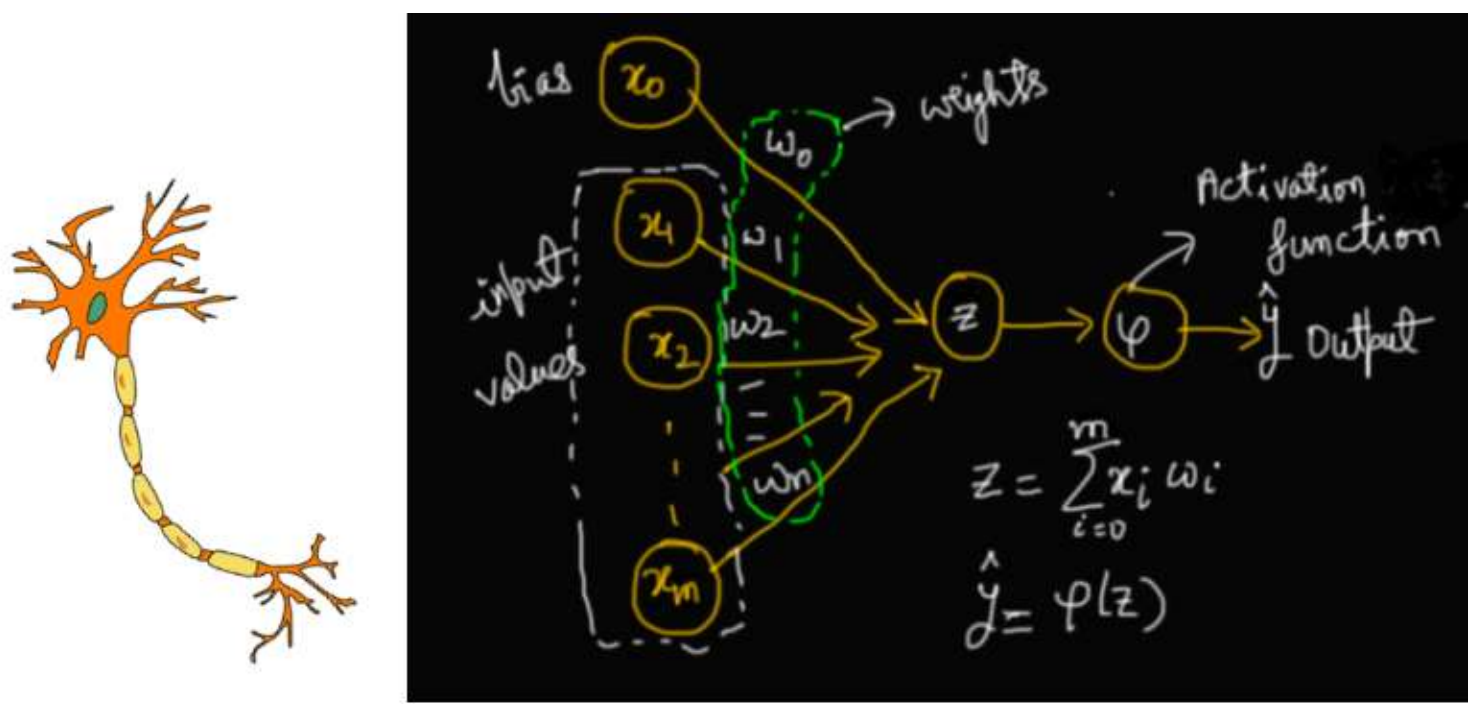

Figure 1: Represents spiking neuron models that generates sharp electrical potential across cell membrane 


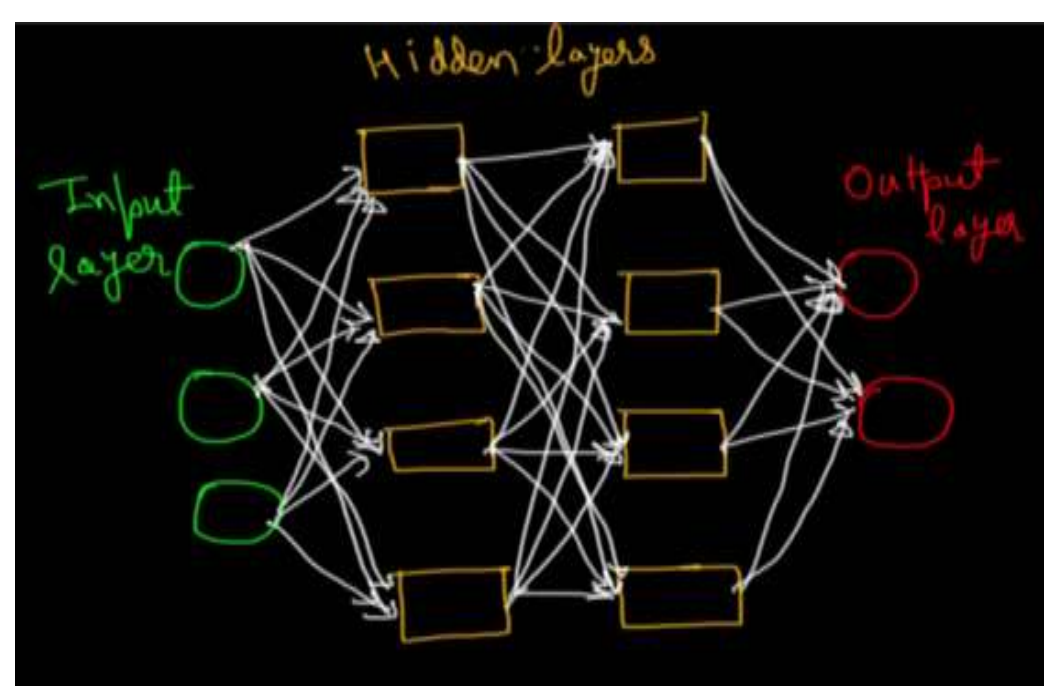

Figure 2 : FFN with 2 hidden layers

A bunch of neuron layers can be utilized to make a neural organization. The organization engineering contrasts dependent on the target it needs to accomplish. A typical organization design is a Feed Forward Neural Network. Neurons are organized directly with no cycles to shape a FFN. It is called feed forward on the grounds that data goes the forward way inside the organization, first through the info neurons layer, then, at that point through the secret neuron layers, and the yield neurons layer .

Any regulated AI model, the FFN should be prepared utilizing marked information. The preparation is through advancing the boundaries by lessening the mistake between the yield esteem and the genuine worth. One such significant boundary to upgrade is the weight every neuron provides for every one of its feedback signals. For a solitary neuron, the weight can be effortlessly processed utilizing the mistake.

Notwithstanding, when a bunch of neurons are gathered in different layers, it is trying to improve the neuron loads in various layers dependent on the blunder processed at the yield layer. The backpropagation calculation assists with resolving this issue [6]. Backpropagation is an old method which goes under the part of PC polynomial math. Here, programmed separation is utilized to compute the slope that is required in the computation of the loads to be utilized in the organization.

As feed forward network [15], in view of actuation of each connected neuron, the yield is gotten. The mistake is spread layer by layer. In view of the rightness of the yield with the ultimate result, the mistake is determined. This mistake is then thus back proliferated to fix blunders of inner neurons. For every information occurrence, the boundaries are advanced by going through various cycles.

\subsection{Network function traffic policies}

The essential objective of digital assaults is to take the endeavor client information, deals information, licensed innovation archives, source codes and programming keys. The foes exhilarate the taken information to distant workers in encoded traffic alongside the normal traffic. 


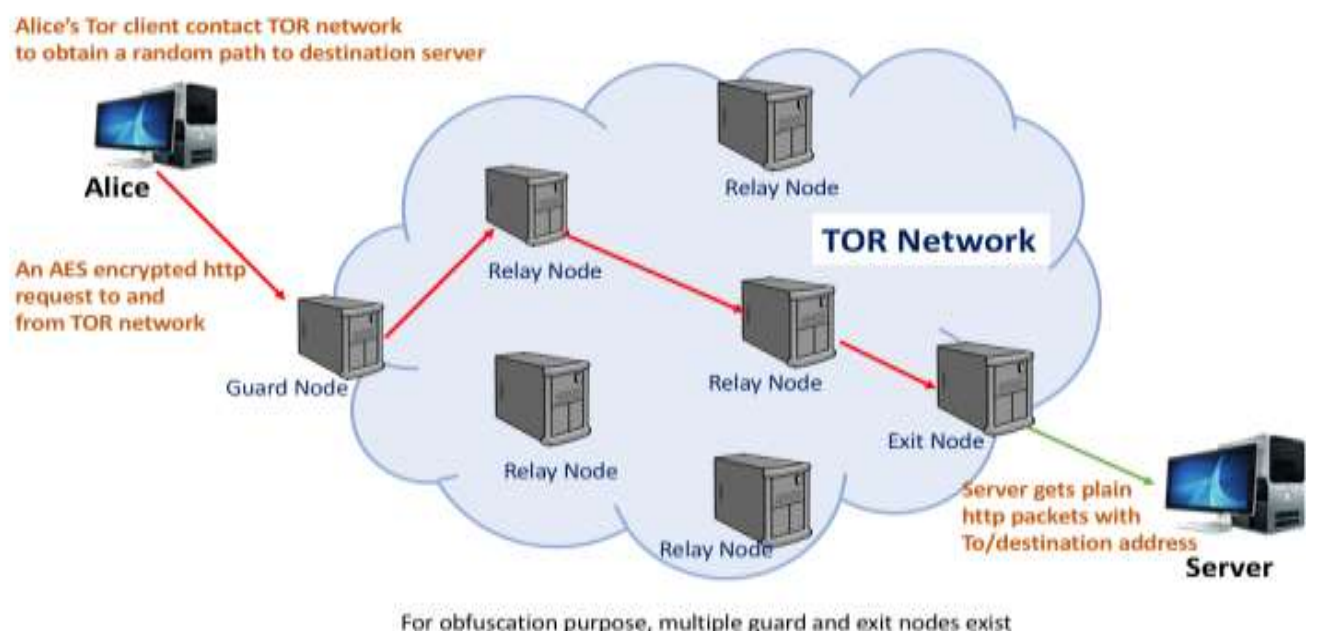

Figure 3: Represents The Onion Router communication interaction of alice and end server. The onion router network makes channel for advanced encryption standard

Frequently enemies utilize an unknown organization that makes it hard for the security safeguards to follow the traffic. Besides, the exhilarated information is normally scrambled, delivering rule-based organization interruption devices and firewalls to be incapable. As of late, unknown organizations have additionally been utilized for $\mathrm{C} \& \mathrm{C}$ by explicit variations of ransomware/malware. For example, Onion Ransomware [7] utilizes the TOR organization to speak with its C\&C.

Among them, TOR is one of the more well known decisions. Pinnacle is a free programming that empowers mysterious correspondence over the web through a particular directing convention known as the onion steering convention [9]. The convention relies upon diverting web traffic over different uninhibitedly facilitated transfers across the world. During the handoff, similar to the layers of an onion strip, every HTTP parcel is scrambled utilizing the public key of the collector.

At every recipient point, the bundle can be decoded utilizing the private key. Upon unscrambling, the following objective hand-off address is uncovered. This carries on until the leave hub of the TOR network is met, where the unscrambling of the bundle closes, and a plain HTTP parcel is sent to the first objective worker. A model directing plan among Alice and the worker is portrayed in the above Figure 3 for delineation.

The first plan of dispatching TOR was to shield the security of clients. In any case, enemies have seized the great Samaritan objective to utilize it for different detestable means all things considered. Starting at 2016, around $20 \%$ of the Tor traffic represents criminal operations. In an undertaking organization, TOR traffic is curtained by not permitting the establishment of the TOR customer or impeding the Guard or Entry hub IP address.

In any case, there are various means through which enemies and malware can gain admittance to the TOR organization to move information and data. The IP impeding methodology is certainly not a sound procedure. Enemies can generate various IPs to do the correspondence. An awful bot scene report by distil networks [5] shows that 70\% of mechanized assaults in 2015 utilized numerous IPs, and $20 \%$ of computerized assaults utilized more than 100 IPs.

Pinnacle traffic can be recognized by examining the traffic bundles. This examination can be on the TOR hub, or in the middle of the customer and the passage hub. The investigation is done on a solitary progression of parcel. Each stream establishes a tuple of source address, source port, objective location, and objective port. 
Organization streams for various time stretches are extricated and examination is carried on them. G. He et al. in their paper "Surmising Application Type Information from Tor Encrypted Traffic" separated burst volumes and bearings to make a HMM model to recognize the TOR applications that may be creating that traffic. The greater part of the mainstream works in this space influence time sensitive elements alongside different elements like size and port data to recognize TOR traffic.

\section{Methodology}

\subsection{Traffic Onion Router}

We take motivation from Habibi et al's " Characterization of Tor Traffic utilizing Time based Features" paper and follow a time sensitive methodology over removed organization stream to recognize TOR traffic for this article. Nonetheless, our engineering utilizes a plenty of other meta-data that can be gotten to arrange the traffic. This is innately because of the Deep Learning engineering that has been decided to tackle this issue.

Table 1: Structural Data Parameters

\begin{tabular}{ll}
$\begin{array}{l}\text { Meta-Information } \\
\text { parameter }\end{array}$ & Parameter Explanation \\
\hline FIAT & $\begin{array}{l}\text { Forward Inter Arrival Time, the time between two packets sent forward direction } \\
\text { (mean, min, max, std). }\end{array}$ \\
\hline BIAT & $\begin{array}{l}\text { Backward Inter Arrival Time, the time be- tween two packets sent backwards } \\
\text { (mean, min, max, std). }\end{array}$ \\
\hline FLOWIAT & $\begin{array}{l}\text { Flow Inter Arrival Time, the time between two packets sent in either direction } \\
\text { (mean, min, max, std). }\end{array}$ \\
\hline ACTIVE & $\begin{array}{l}\text { The amount of time time a flow was active before going idle (mean, min, max, } \\
\text { std). }\end{array}$ \\
\hline IDLE & $\begin{array}{l}\text { The amount of time time a flow was idle before becoming active (mean, min, max, } \\
\text { std). }\end{array}$ \\
\hline FB PSEC & $\begin{array}{l}\text { Flow Bytes per second. Flow packets per second. duration: The duration of the } \\
\text { flow. }\end{array}$
\end{tabular}

An example of A sequence of invertible transformations of network load and rate at which error occurs

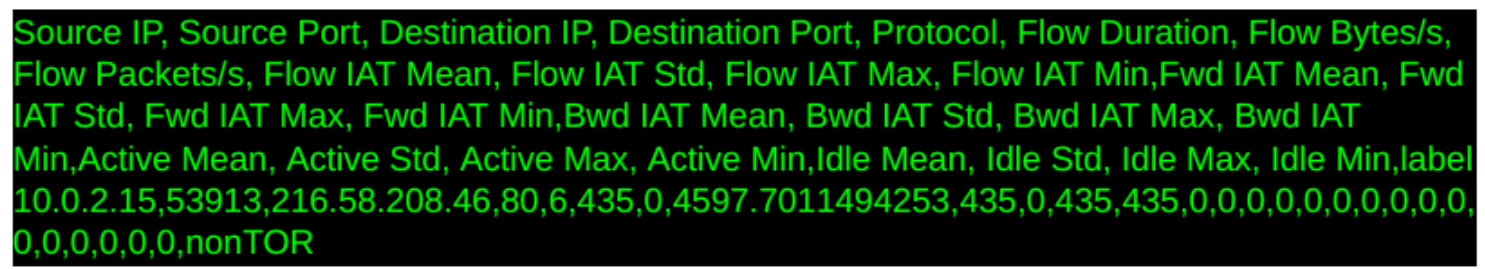

Figure 4 : Represents prototype of training information set 
Kindly note that source Internet Protocol /port and objective IP/port, alongside the convention field, have been eliminated from the case as they overfit the model. We measure any remaining components utilizing a profound feed forward neural organization with $\mathrm{N}$ covered up layers. The engineering of the neural organization is displayed in picture 5

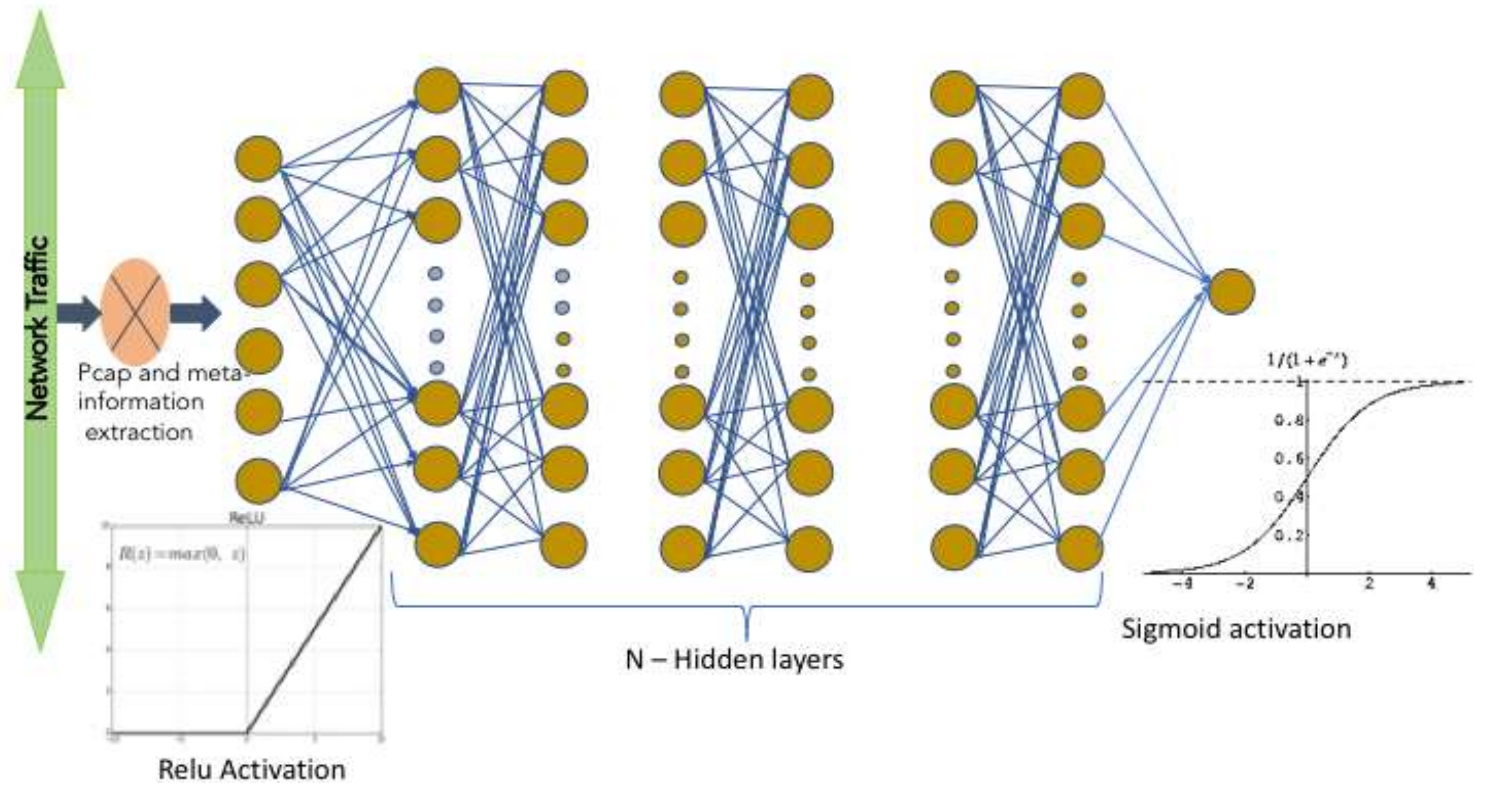

Figure 5 : DL Network representation used in Traffic onion router foundation

Table 2 : The Machine Learning and Deep Learning for Traffic Detection

\begin{tabular}{llll} 
Classifier used & Precision & Recall & F-Score \\
Logistic Regression & 0.87 & 0.87 & 0.87 \\
SVM & 0.9 & 0.9 & 0.9 \\
\hline Naìve Bayes & 0.91 & 0.6 & 0.7 \\
Random Forest & 0.96 & 0.96 & 0.96 \\
Deep Learning & 0.95 & 0.95 & 0.95
\end{tabular}

Accuracy

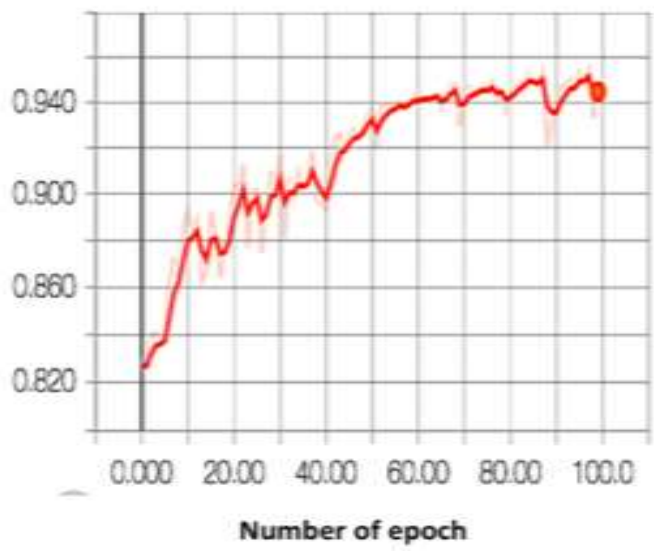

Loss

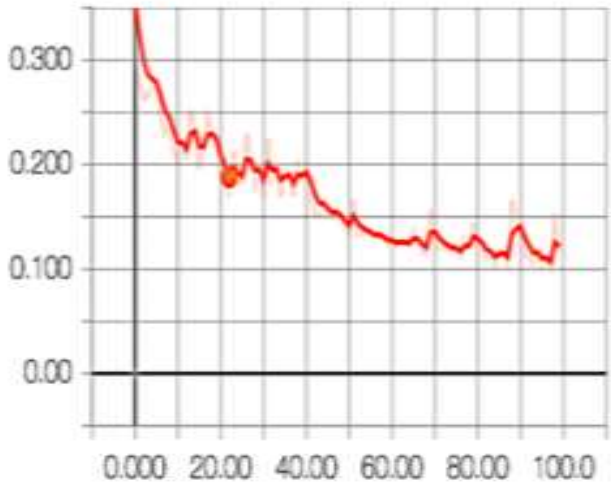

Number of epoch

Figure 6 : Measurements and Visualization statics of machine learning work flow 
The profound learning framework was contrasted and different assessors. Standard order measurements of Recall, Precision and F-Score were utilized to quantify the adequacy of the assessors. Our Deep learning -based framework had the option to distinguish the TOR class well. In any case, it is the Non-Tor class that we need to give more significance to. It is seen that a Deep Learning-based framework can decrease the bogus positive cases for Non-Tor classification.

Among different classifiers, Random Forest and Deep learning based methodologies perform better compared to the rest. The outcome shown depends on 55,000 preparing occasions. The dataset utilized in this test is nearly more modest than run of the mill DL-based frameworks. As the preparation information builds, execution would increment further for both DL-based and Random woodland classifier.

Notwithstanding, for huge datasets, a Deep Learning -based classifier normally beats different classifiers, and it very well may be summed up for comparative sorts of uses. For instance, on the off chance that one requirements to prepare a classifier to distinguish the application utilized by TOR, then, at that point just the yield layer needs retraining, and the wide range of various layers can be kept something similar. While other Machine Learning -classifiers should be retrained for the whole dataset. Remember that retraining the model might take critical registering assets for enormous datasets.

\section{Conclusion:}

Anonym zed traffic location is a nuanced challenge that each undertaking faces. The foes use TOR directs to infiltrate information in mysterious mode. Current methodologies by peak traffic recognition sellers rely upon obstructing known section hubs of the TOR organization. This is certifiably not a versatile approach and can be effortlessly skirted. A conventional strategy is to utilize profound learning-based strategies.

In this article, we introduced a profound learning-based framework to identify the TOR traffic with high review and exactness. Tell us your interpretation of the present status of profound learning, or then again in the event that you have any substitute methodologies, in the remarks area underneath.

\section{Acknowledgments}

I would like to acknowledge the authors for the review of my paper and supporting the research and we would like to thank Dr. Y. Madhavee Latha ,Principal- Malla Reddy Engineering College for Women(UGC Autonomous Campus), Maisammaguda, Dhulapally, Kompally, Medchal (M), Secunderabad, Telangana, India . Furthermore, the authors would like to thank all colleagues and friends in the Computer Science Department for their support.

\section{REFERENCES}

\section{Conferences:}

[1]: Quamar Niyaz, Weiqing Sun, Ahmad Y Javaid, and Mansoor Alam, "A Deep Learning Approach for Network Intrusion Detection System," IEEE Transactions on Emerging Topics in Computational Intelligence, 2018.

[2]: Daniel Gibert, "Convolutional Neural Networks for Malware Classification," Thesis 2016.

[3]: Wookhyun Jung, Sangwon Kim, Sangyong Choi, "Deep Learning for Zero-day Flash Malware Detection," IEEE security, 2017.

[4]: Pawet Kobojek and Khalid Saeed, "Application of Recurrent Neural Networks for UserVerification based on Keystroke Dynamics, "Journal of telecommunications and information technology, 2016.

[5]:Deep Learning Security Papers, http://www.covert.io/the-definitive-security-datascience-andmachinelearning-guide/\#deep-learning-and-security-papers, accessed on May 2018.

[6]: "Deep Learning," Ian Goodfellow, Yoshua Bengio, Aaaron Courville; pp 196, MIT Press, 2016.

[7]: "The Onion Ransomware," https://www.kaspersky.co.in/resource-center/threats/onion-ransomwarevirus-threat, Retrieved on November 29, 2017. 
[8]: "5 best alternative to TOR.," https://fossbytes.com/best-alternatives-to-tor-browser-to-browseanonymously/, Retrieved on November 29,2017.

[9]: Tor. Wikipedia., https://en.wikipedia.org/wiki/Tor_(anonymity_network), Retrieved on November 24, 2017.

[10]: He, G., Yang, M., Luo, J. and Gu, X., “Inferring Application Type Information from Tor Encrypted Traffic," Advanced Cloud and Big Data (CBD), 2014 Second International Conference on (pp. 220-227), Nov. 2014

[11]: Habibi Lashkari A., Draper Gil G., Mamun M. and Ghorbani A., "Characterization of Tor Traffic using Time based Features," Proceedings of the 3rd International Conference on Information Systems Security and Privacy - Volume 1, pages 253-262, 2017.

[13]: Juarez, M., Afroz, S., Acar, G., Diaz, C. and Greenstadt, R., "A critical evaluation of website fingerprinting attacks, " Proceedings of the 2014 ACM SIGSAC Conference on Computer and Communications Security (pp. 263-274), November 2014

[14]: Bai, X., Zhang, Y. and Niu, X., “Traffic identification of tor and web-mix," Intelligent Systems Design and Applications, ISDA'08. Eighth International Conference on (Vol. 1, pp. 548-551). IEEE, November 2008

\section{Journal}

[15] Venkatakrishna Reddy, Dr S Pradeep, “Envision Foundational of Convolutional Neural Network “, IJITEE , Vol 10, Issue 06, pages -54-60 